\title{
Multi-Wavelength View of Supernova Remnants
}

\author{
Manami Sasaki ${ }^{1}$ \\ ${ }^{1}$ Institute for Astronomy and Astrophysics, Eberhard Karls University Tübingen, Sand 1, D-72076 Tübingen, Germany \\ Corresponding author: sasaki@astro.uni-tuebingen.de
}

\begin{abstract}
This contribution gives a very short overview on the emission of supernova remnants and the processes that are responsible for both the thermal and non-thermal origins of the emission, typically observed in radio, X-rays, and up to $\gamma$-rays. We discuss in particular the case of the Galactic SNR CTB 109. As detailed X-ray studies combined with observations in radio have shown, CTB 109 is interacting with a giant molecular cloud complex. The interaction of the SNR shock with dense interstellar clouds is responsible for both the unusual semi-circular morphology of the SNR and the bright X-ray feature inside the SNR, and, as has been shown recently, seems also to play a major role in the production of $\gamma$-rays.
\end{abstract}

Keywords: supernova remnants - radio - IR - optical - X-rays - $\gamma$-rays.

\section{Introduction}

Supernova remnants (SNRs) are objects that are formed by a supernova (SN) explosion at the end of the life of a star. Owing to the shock waves of the SN explosion, the stellar ejecta as well as the ambient medium is heated to temperatures higher than a million Kelvin. Therefore, SNRs are responsible for the heating of the interstellar medium (ISM) and the distribution of heavier elements that were processed inside a star. In addition, both electrons and nuclei can be accelerated in the shock waves of the SNRs to relativistic energies. Therefore, SNRs are also considered to be one source of Galactic cosmic rays.

By now, about 280 SNRs are known in our Galaxy and about 50 in the Magellanic Clouds. Detailed studies of SNRs in the Galaxy and the Magellanic Clouds allow us to understand the nature of their shocks, the various radiation processes that are responsible for the complex emission observed over the full electromagnetic spectrum from radio to $\gamma$-rays, the interaction of the shock with the cooler, and often inhomogeneous ISM, and the acceleration of particles.

\section{SNRs at Lower Energies}

\subsection{Radio emission}

In the strong shock waves of SNRs, electrons are accelerated and interact with the interstellar magnetic fields. These electrons emit synchrotron radiation and thus make the SNR bright in radio.

The distribution of the surface brightness of the electron synchrotron emission in SNRs can be obtained from radio imaging observations. In general, the radio emission consists of narrow shell-like or filamentary emission and additional diffuse emission. The reason for these two kinds of synchrotron emission is that particles with higher energies lose their energy faster through synchrotron emission than those with lower energies. The long-lived lower-energy particles can diffuse further away from the shock and radiate over a much wider area, producing the more extended diffuse emission with a steeper spectrum.

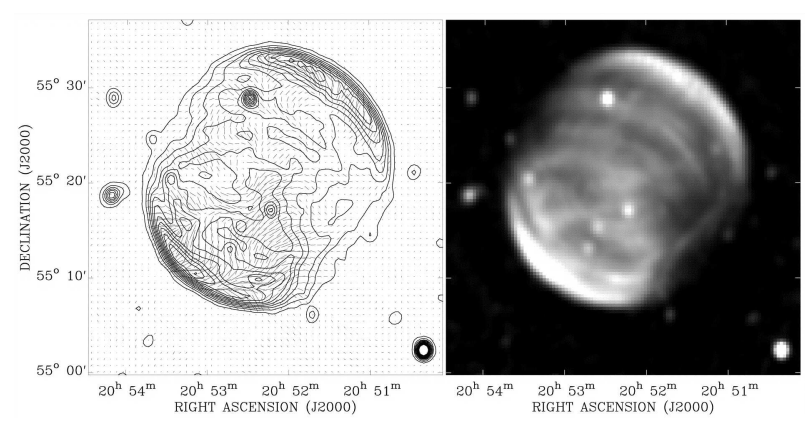

Figure 1: Radio continuum emission from SNR DA $530(1420 \mathrm{MHz})$ in contour representation (left) and gray scale (right). Vectors in the left image show the polarized intensity by their length and the direction of the E-field by their orientation (Landecker et al., 1999).

Since synchrotron emission is polarized, radio observations can reveal the magnetic field structure in and around SNRs. Observations have shown that young remnants in general have a radial field structure. For remnants in the free expansion phase and in the early phases of the adiabatic expansion, radial motions due 
to Rayleigh-Taylor instabilities at the contact discontinuity between the shocked ejecta and the shocked ISM can enhance the radial magnetic fields. On the contrary, older remnants have a more tangential field structure with higher polarized fractions (Milne, 1987). The polarized fraction in younger remnants are typically $p=10-15 \%$, while older remnants like SNR DA 530 can have a polarized fraction of $p=50 \%$ (Fig. 1 , Landecker et al., 1999).

\section{Thermal X-Ray Emission}

The outward moving shock wave of an SNR heats the interstellar medium, while the reverse shock that propagates inward heats the ejecta of the SN explosion. As a whole the ionized gas inside an SNR has temperatures of $\sim 10^{6-7} \mathrm{~K}$ typically.

In plasmas the excitation and ionization of the atoms are mainly induced by electrons that collide with ions. SNRs have very low densities and thus are optically thin. They can be considered to be out of thermal and ionization equilibrium, especially when they are still young. If the time after the plasma has been shocked is short, only a few collisions have occurred that cause ionization. The ionization states of the ions at a given temperature are thus lower than in the case of collisional ionization equilibrium (CIE). Instead, the plasma in SNRs is characterized by non-equilibrium ionization (NEI). The shape of the continuum of the thermal plasma is primarily determined by the electron temperature, while the temperature of ions might be different than that of electrons. The line emission in the X-ray band can be used to derive the ionization states of the ions as well as the element abundances. Therefore, the X-ray spectrum can, on the one hand, reveal the element distribution in the ejecta, and on the other hand, the typical abundances of the ISM in which the SNR expands.

\subsection{Charge exchange}

Newer observations of SNRs in X-rays have revealed spectral features indicating charge exchange (CX) between the hot thin plasma of an SNR and the cold interstellar gas. X-ray emission from CX can in principle be produced at any astrophysical site where hot plasma interacts with (partially) neutral gas, and was originally discoverd in X-ray data of comets (Lisse et al., 1996, Cravens, 1997). First observational evidences of CX in SNRs had been obtained as a broad component of $\mathrm{H} \alpha$ emission (e.g., Chevalier et al., 1980). Observations of the rim of the Cygnus Loop using Suzaku and XMMNewton have revealed a significant emission feature at $0.7 \mathrm{keV}$, which was identified to be a complex of He-like $\mathrm{O} \mathrm{K}(\gamma+\delta+\epsilon)$ and was interpreted as CX emission from interaction between He-like $\mathrm{O}$ and neutrals (Fig. 2, Katsuda et al., 2011).

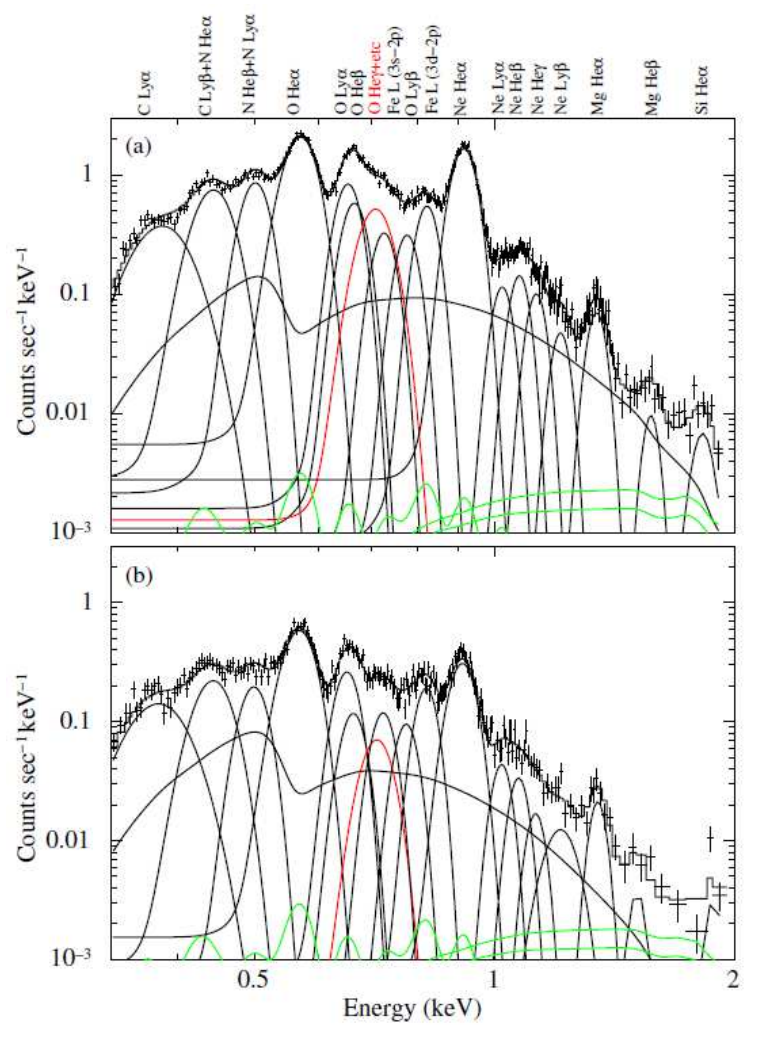

Figure 2: Non-X-ray background subtracted spectrum of a northeastern region of the Cygnus Loop SNR taken with Suzaku along with the best-fit model consisting of a bremsstrahlung plus Gaussians (Katsuda et al., 2011). Line identifications are given on top of the upper panel. The Gaussian shown in red represent the CX emission. The X-ray background components are shown in green.

\subsection{Overionization}

New Suzaku observations of mixed-morphology (MM) SNRs in the last few years have shown that in many cases the thermal X-ray emission can be interpreted as that from an overionized, recombining plasma in ionization non-equilibrium (e.g., Yamaguchi et al., 2009, see Fig. 3). Mixed-morphology SNRS, also called thermalcomposite remnants, are usually found in dense regions near molecular clouds and show very little or no shell emission. The X-ray emission is bright in the (projected) interior and is of thermal nature. The signature of an overionized plasma is a radiative recombination continuum (RRC) and strong Ly emission of, e.g., Si, $\mathrm{S}$, or Fe.

In general, SNRs are underionized and the shocked plasma slowly develops to ionization equilibrium. Therefore, it is rather surprising to observe overion- 
ization in SNRs. To reach overionization in a plasma above $\sim 10^{6} \mathrm{~K}$, electrons have to cool rapidly. Possible processes for such a cooling are either fast expansion, thermal conduction, or radiative cooling with enhanced abundances, which are all processes that are likely to occur in particular in SNRs evolving in significantly inhomogenous ISM.

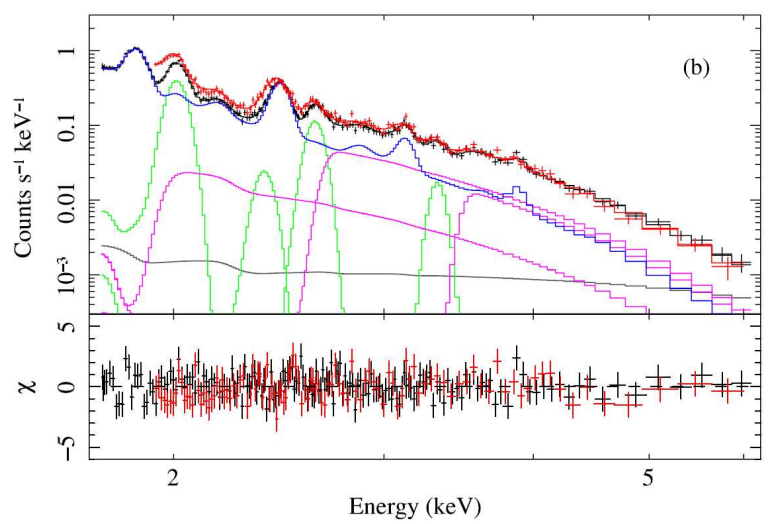

Figure 3: Suzaku XIS spectrum in the $1.75-6.0 \mathrm{keV}$ band (black: FI, red: BI). The best fit model is shown including the CXB (black) and VAPEC and Gaussians for the source emission. RRC emisison of $\mathrm{H}$-like $\mathrm{Mg}$, Si, and $\mathrm{S}$ are shown in magenta (Yamaguchi et al., 2009).

\section{SNRs as Particle Accelerators}

\subsection{Non-thermal X-ray emission}

Supernova remnants are non-thermal, extended radio sources with a flux spectrum described by a power-law with $S_{\nu} \propto \nu^{\alpha}$ with $\alpha \approx-0.5$. The synchrotron radiation is emitted by a non-thermal population of relativistic electrons, which can be described by a powerlaw. Synchrotron emission in radio indicates that there are electrons with energies in the $\mathrm{GeV}$ range in the SNR shell. Synchrotron emission from an SNR in the X-ray band was confirmed for the first time for the remnant of SN 1006 (Koyama et al., 1995) based on $A S C A$ data, which showed that the non-thermal emission is dominating the emission of two outer shell regions. Soon after, more SNRs were also confirmed to radiate non-thermal X-ray emission: Cas A (e.g., The et al., 1996, Allen et al., 1997), RX J1713.7-3946 (G347.30.5, Pfeffermann \& Aschenbach, 1996), RX J0852.04622 (G266.2-1.2, Vela Jr, Aschenbach 1998). The Xray emission of RX J1713.7-3946 and RX J0852.0-4622 is completely of non-thermal nature with no thermal emission detected so far. X-ray synchrotron emission is only observed in younger SNRs and is indicative of electrons with energies of up to $10^{14} \mathrm{eV}$.
The angular resolution of the newer X-ray telescopes, in particular that of the Chandra X-ray observatory, made it possible to reveal narrow filaments of non-thermal X-ray emission close to the outer shock in several young SNRs: SN 1006, RX J1713.7-3946, RX J0852.0-4622, Cas A, Tycho, and Kepler. These filaments are very thin $\left(\sim 1-2^{\prime \prime}\right)$ and indicate relatively high magnetic fields $(B=100-600 \mu \mathrm{G})$. Recently, a deep Chandra observation of Tycho's SNR revealed bright non-thermal stripes, clearly seen in the energy band of $>4 \mathrm{keV}$ (Fig.4, Eriksen et al., 2011). The stripes are likely caused by peaks in magnetic fields.

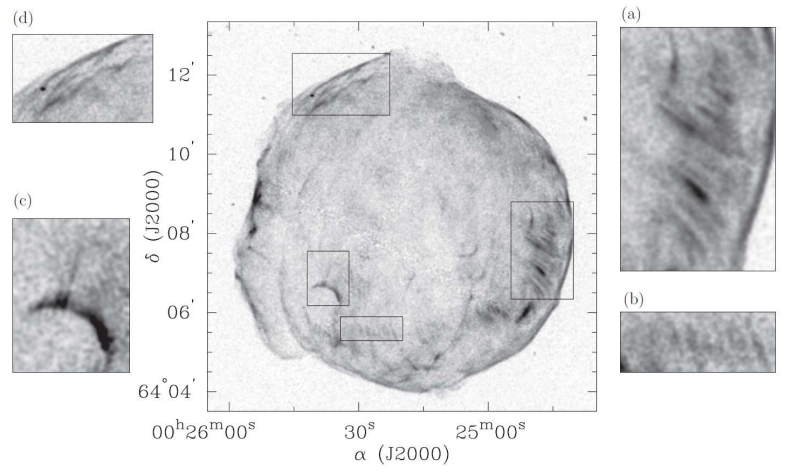

Figure 4: Chandra image of the Tycho's SNR in the energy band of $4.0-6.0 \mathrm{keV}$. The boxes show the regions with stripes or filaments of non-thermal emission (Eriksen et al., 2011).

\subsection{Gamma-ray emission}

In the last decade some SNRs have been detected with $\gamma$-ray observatories and are now well established astrophysical $\gamma$-sources. There are two major origins of $\gamma$-ray emission in SNRs:

- Leptonic: Interactions of relativistic electrons with low-energy photons of the cosmic microwave background cause inverse Compton up-scattering of the photons. In addition, the non-thermal electrons will also emit bremsstrahlung through interactions with ions in the SNR.

- Hadronic: Accelerated nuclei can produce $\gamma$ ray emission if they collide with atomic nuclei, thereby creating, among others, neutral $\pi^{0} \mathrm{~s}$, which decay into $\gamma$-ray photons. This is believed to be enhanced in SNRs near high-density clouds where the probability for the collision of the accelerated protons from the SNR with cold protons or nuclei is higher (e.g., IC 443, W44, see Fig. 5).

The $\mathrm{GeV}$ and $\mathrm{TeV}$ emission of SNRs can thus give us a direct view of the accelerated particles. 

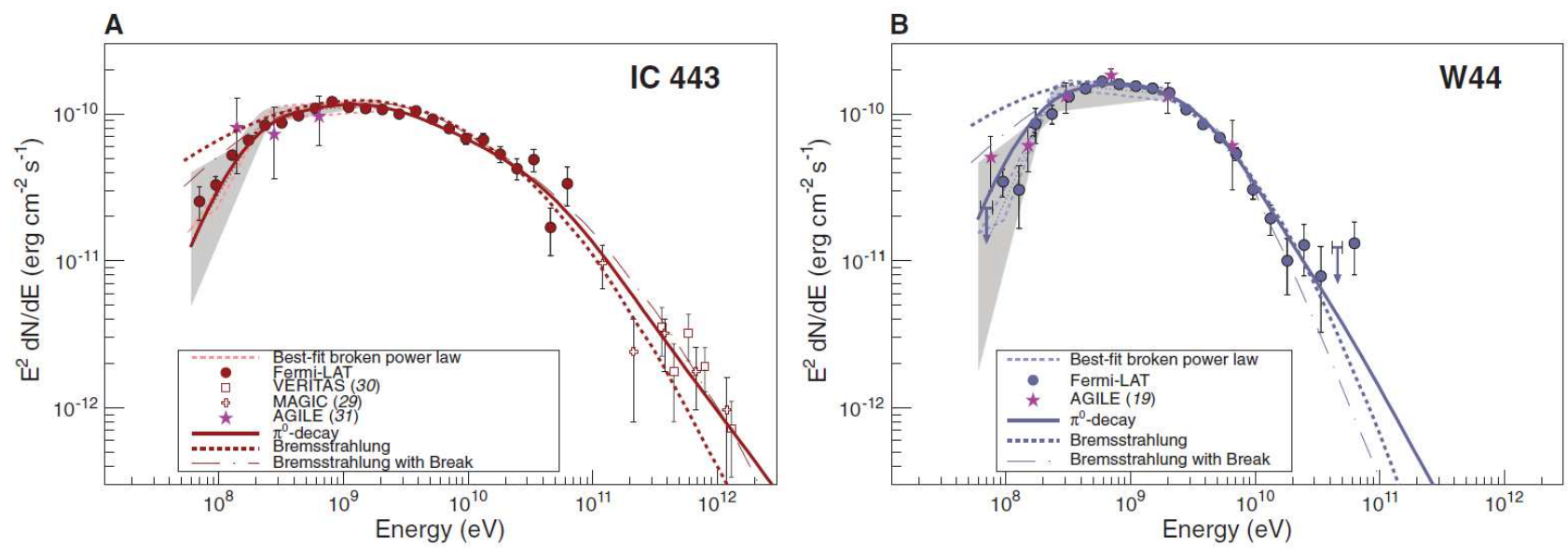

Figure 5: Gamma-ray spectra of the SNRs IC 443 and W44 and fitted models (Ackermann et al., 2013).

\section{The Galactic SNR CTB 109}

The Galactic supernova remnant CTB 109 (G109.1$1.0)$ is the host of the anomalous X-ray pulsar (AXP) 1E 2259+586 (Fahlman \& Gregory, 1981) and has an interesting semi-circular morphology in both the X-ray and the radio. It is believed to be located next to a giant molecular cloud (GMC) complex and is thus one of the most striking examples of an interaction of an SNR with a molecular cloud.

The SNR was discovered in X-rays with Einstein by Gregory \& Fahlman (1980). Hughes et al. (1981) identified the radio counterpart in the Galactic plane survey at $49 \mathrm{~cm}$ with the Westerbork Synthesis Radio Telescope. Its shell is incomplete on the western side both in X-rays and in radio. The semi-circular radio and X-ray morphology of CTB 109 suggests the shock has been stopped by the GMC complex in the west. An elongated feature in $\mathrm{CO}$ ('CO arm') extends from the GMC complex to an X-ray minimum inside the SNR in the north (Tatematsu et al., 1987), indicating that a part of the dense gas of the GMC complex extends in front of the remnant.

We performed observations of the entire SNR CTB 109 with XMM-Newton using the European Photon Imaging Cameras (EPICs). A mosaic of all observations is shown in Fig. 6. As mentioned before, CTB 109 seems to have expanded into a dense medium in the west, while the shock wave is expanding into a lowerdensity medium in the east. The radii of the northern and the southern periphery of CTB 109 are slightly smaller than to the east suggesting that CTB 109 is confined by denser material also in the north and the south. However, the relative difference is only $\sim 5-$ 10\%. Therefore, the overall shape of the shell can be considered as a semi-sphere.

There is an X-ray bright interior region known as the Lobe, which is brighter than any part of the SNR shell. The X-ray spectrum from the Lobe obtained with XMM-Newton is completely thermal (Sasaki et al., 2004). We found CO clouds around the Lobe in new high-resolution $\mathrm{CO}$ data from the Five College Radio Observatory (Sasaki et al., 2006). One of the CO clouds overlaps with the Lobe and has an additional component towards higher negative velocities in the velocity profile. We believe that the bright $\mathrm{X}$-ray emission of the Lobe is the result of the interaction of the SNR shock with a dense molecular cloud, which was hit by the blast wave on the western side. The main part of the cloud was heated and is now visible as the Lobe, while some denser parts might have survived.

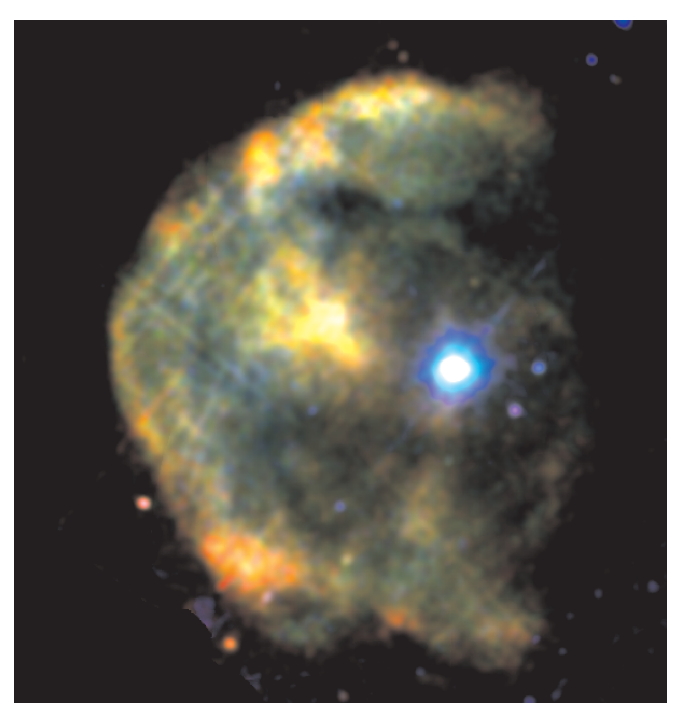

Figure 6: Mosaic RGB image of the SNR CTB 109 created from the XMM-Newton observations: red $=0.3$ $-0.9 \mathrm{keV}$, green $=0.9-1.5 \mathrm{keV}$, blue $=1.5-4.0 \mathrm{keV}$ (Sasaki et al., 2004). 

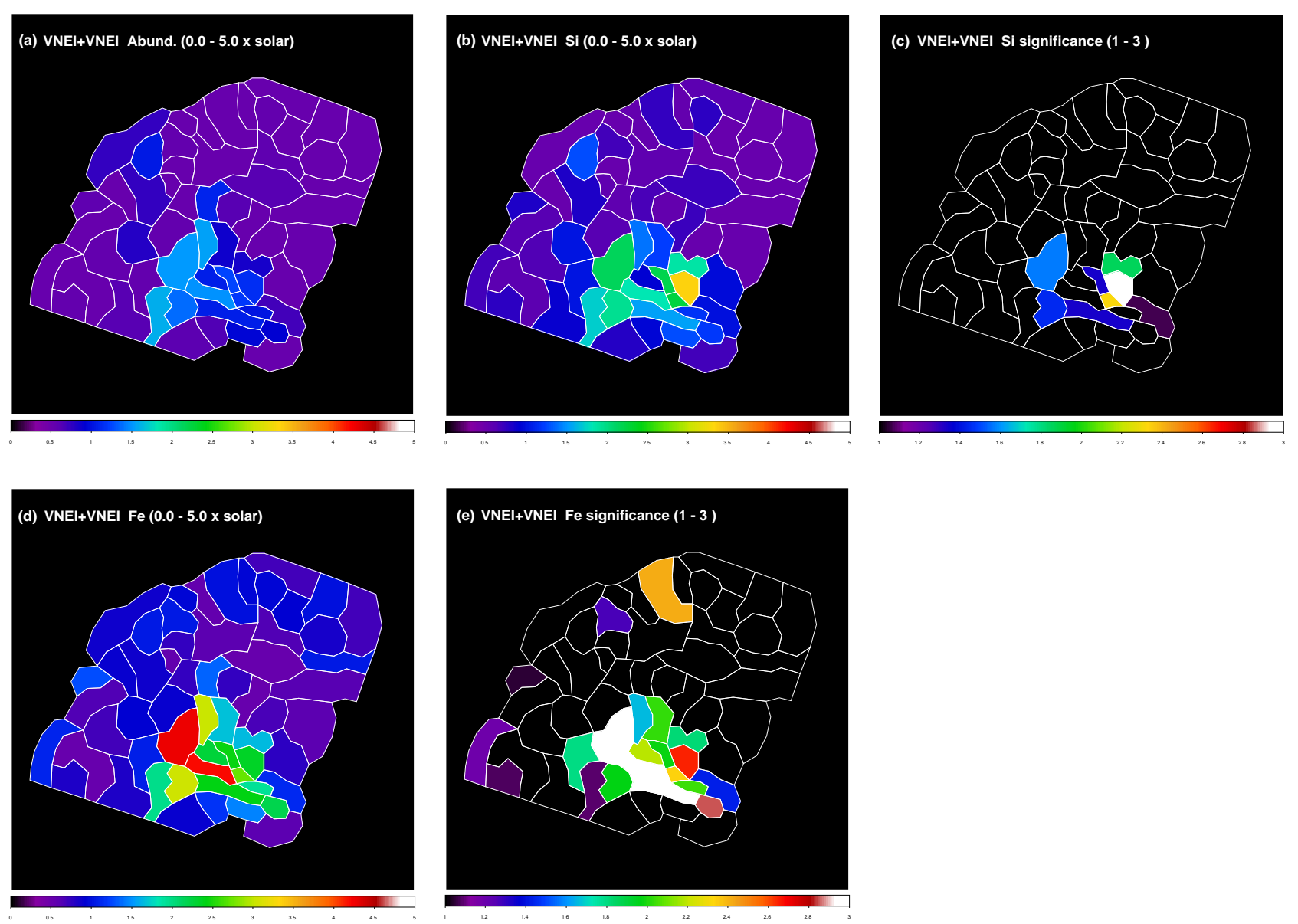

Figure 7: Best fit abundances obtained for the emission of SNR CTB 109 from the analysis of the Chandra ACIS spectra by assuming a thermal model that consists of two VNEI components for thermal non-equilibrium plasma for the shocked ISM and for the shocked ejecta (Sasaki et al., 2013). The shown parameters are: abundances for elements other than Si, S, and Fe fitted for the ejecta emission (a), Si abundance fitted for the ejecta emission (b) and its significance calculated as (abundance of the $\mathrm{Si}$ - abundance of the other elements)/error of the abundance of $\mathrm{Si}(\mathrm{c}), \mathrm{Fe}$ abundance and its significance (d and e, respectively).

Through the analysis of a deep, high-resolution data of the region around the Lobe taken with Chandra Advanced CCD Imaging Spectrometer (ACIS), we discovered ejecta emission inside the SNR west of the Lobe (Sasaki et al., 2013). The distriubtion of the Siabundance and Fe-abundance are shown in in Fig. 7. These images show the parameter values obtained from the analysis of the spectra extracted in the regions indicated. The abundances measured for the ejecta emission are all comparable to or lower than solar values, except for the Lobe. Here, the Si and Fe abundances are in particular significantly higher. The enhanced abundances in the Lobe suggest that there is an ejecta clump or a conglomeration of an ejecta clump and a shocked cloud.

At the position of CTB 109 a GeV source was detected with Fermi (Castro et al., 2012, see Fig. 8). This $\gamma$-ray source is located southwest of the Lobe where the SNR appears dark in X-rays and thus is most likely highly absorbed. Castro et al. (2012) performed a comprehensive modeling of the emission of CTB 109, in which they could reproduce the fluxes in radio, X-rays, and $\gamma$, as well as the $\mathrm{X}$-ray spectrum taken with the $X M M$-Newton EPIC in a model including a significant contribution by $\pi^{0}$-decay. This is again consistent with the picture that there is significant interaction between the SNR shock and a dense interstellar cloud.

\section{Summary}

Being driven by strong shock waves formed in supernova explosions, supernova remnants are one of the main drivers of the matter cycle and dynamic evolution of galaxies. They are responsible for the chem- 
ical enrichment of galaxies. The shock waves create new structures in the ISM by forming a cavity filled with hot thin gas and sweep the colder gas around it. Particles are accelerated in the shock waves and their emission is observed over the entire range of the observable electromagnetic spectrum. In recent years observations of X-ray synchrotron radiation confirmed the existence of electrons with energies in the $\mathrm{TeV}$ range in SNR shocks. Newest $\mathrm{GeV}$ and $\mathrm{TeV}$ obervations indicate inverse Compton scattering as well as pion-decay processes in and around SNR shocks. Self-consistent modeling of the spectrum from radio to $\mathrm{TeV}$ as in the case of the Galactic SNR CTB 109 helps us to understand both the heating and acceleration processes in SNR shocks.

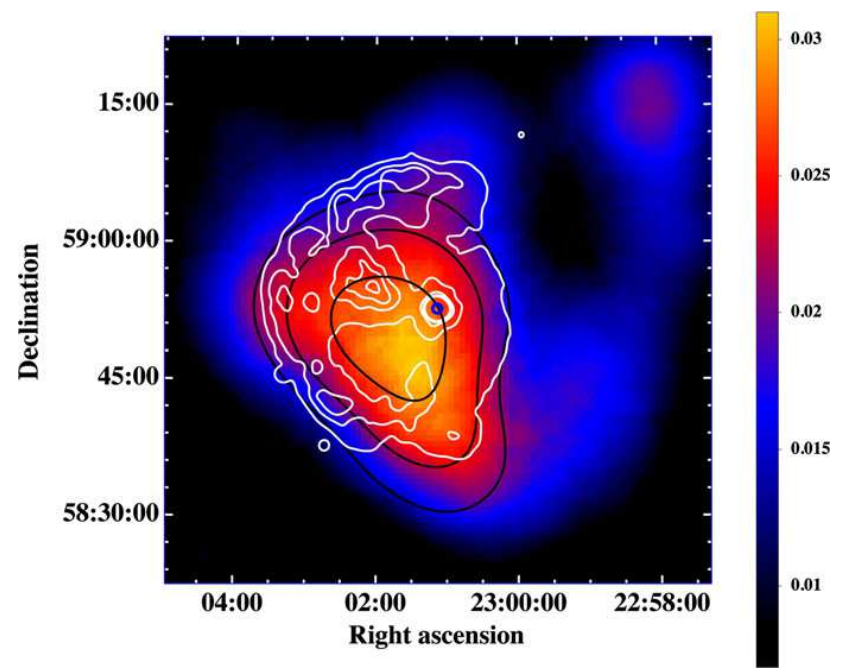

Figure 8: Smoothed Fermi-LAT count maps in the range $2-200 \mathrm{GeV}$ (Castro et al., 2012). White contours show the X-ray emission $(0.5-5.0 \mathrm{keV})$ from XMM-Newton data.

\section{Acknowledgement}

M.S. acknowledges support by the Deutsche Forschungsgemeinschaft through the Emmy Noether Research Grant SA 2131/1.

\section{References}

[1] Ackermann, M., et al. 2013, Science, 339, 807 doi:10.1126/science. 1231160

[2] Allen, G. E., et al. 1997, ApJL, 487, 97

[3] Aschenbach, B. 1998, Nature 396, 141 doi:10.1038/24103

[4] Castro, D., Slane, P., Ellison, D. C., Patnaude, D. J. 2012, ApJ, 756, 88 doi:10.1088/0004-637X/756/1/88
[5] Chevalier, R. A., Kirshner, R. P., Raymond, J. C. 1980, ApJ, 235, 186

[6] Cravens, T. E. 1997, Geophys. Res. Lett., 24, 105

[7] Eriksen, K. A., et al. 2011, ApJL, 728, 28 doi:10.1088/2041-8205/728/2/L28

[8] Fahlman, G. G. \& Gregory, P. C. 1981, Nature, 293, 202 doi:10.1038/293202a0

[9] Gregory, P. C. \& Fahlman, G. G. 1980, Nature, 287, 805 doi:10.1038/287805a0

[10] Hughes, V. A., Harten, R. H., Costain, C. H., van den Bergh, S. 1981, ApJ, 246, L127 doi:10.1086/183568

[11]

[12] Katsuda, S., et al. 2011, ApJ, 730, 24 doi:10.1088/0004-637X/730/1/24

[13] Koyama, K., et al. 1995, Nature, 378, 255 doi:10.1038/378255a0

[14] Landecker, T. L., et al. 1999, ApJ, 527, 866

[15] Lisse, C. M., et al. 1996, Science, 274, 205 doi:10.1126/science.274.5285.205

[16] Milne, D. K., 1987, AuJPh, 40, 771

[17] Pfeffermann, E., Aschenbach, B. 1996, Proc. of 'Röntgenstrahlung from the Universe', 267

[18] Sasaki, M., Kothes, R., Plucinsky, P. P., Gaetz, T. J., Brunt, C. M. 2006, ApJ, 642, L149 doi:10.1086/504844

[19] Sasaki, M., et al. 2004, ApJ, 617, 322

[20] Sasaki, M., Plucinsky, P. P., Gaetz, T. J., Bocchino, F. 2013, A\&A, 552, 45

[21] Tatematsu, K., et al. 1987, A\&A, 184, 279

[22] The, L.-S., et al. 1996, A\&AS, 120, 357

[23] Yamaguchi, H., et al. 2009, ApJL, 705, 6 doi:10.1088/0004-637X/705/1/L6

\section{DISCUSSION}

CARLOTTA PITTORI's Comment: To distinguish between IC and $\pi^{0}$ decay in the $\mathrm{GeV}$ range, at least in some cases like W44, the new Planck data combined with AGILE and Fermi data are very much constraining. The MW picture in the case of middle-aged SNRs interacting with molecular clouds seem to largely favour the hadronic scenario ( $\pi^{0}$ decay). 